\title{
GPS Tracking System Coupled With Image Processing In Traffic Signals to Enhance Life Security
}

\author{
Manoj Prabhakar $\mathrm{K}^{1}$ and Manoj Kumar $\mathrm{S}^{2}$ \\ ${ }^{1}$ Anna University, Chennai, India \\ manojkrishs@gmail.com \\ ${ }^{2}$ Anna University, Chennai, India \\ manojkumars.msec@gmail.com
}

\begin{abstract}
In modern and crowded traffic life, it becomes mandatory to save the human life by reaching the hospital very sooner than possible. This paper explains about the advancement in tracking the ambulance presence amidst the traffic with the usage of GPS tracking system calculating the position of the ambulance from the traffic signal which replaces the RF transmitter receiver circuitry. This paper also retains the use of the image capturing technology available from the base paper (IMPACT OF IMAGE PROCESSING IN SAVING THE HUMAN LIFE BY AUTOMATING TRAFFIC SIGNALS) to identify the presence of the ambulance, thereby changing the signal to green automatically in favour of ambulance.
\end{abstract}

\section{KEYWORDS}

Image Processing, Trigger, Ambulance, Human Life, Ambulance GPS Tracking system, Ambulance position, Proximity sensors.

\section{INTRODUCTION}

An image is nothing but the subset of signal. Generally a signal is used to convey the information from one end to other end. These sorts of signals can be used in many ways. Electrical signals are used in television, radio, etc, which is transmitted by electrical quantities. Here the digital processing is the process of extracting information from the signals. Every signal will have certain information which is to be transmitted to the other end.

The digital signal processing is mainly related representing and processing of the numbers (sequence) and symbols. The signals have many characteristics such as its shapes, time durations, amplitudes and etc. These signals can be classified as continuous and discrete time signals based on sampling. There are two types of signals, analog and digital signals. If the signals are repeating after some period, they are called periodic signals. Every signal can be fixed by Mathematical functions. The signals are classified into three types according to its dimensions. They are 1D (One-Dimensional), 2D (Two-Dimensional), 3D (Three-Dimensional).

The One Dimensional signals will be using time waveforms $\mathrm{x}(\mathrm{t})$ or $\mathrm{f}(\mathrm{t})$. Two dimensional signals will be used two axis such as $(\mathrm{x}, \mathrm{y}) 2 \mathrm{D}$ signals are the function of two independent variables called as $(\mathrm{x}, \mathrm{y})$ and has been projected in $\mathrm{x}, \mathrm{y}$ plane. The 2D signals will be projecting the images and still 
photographs. The Three dimensional signals will be plotted in $(\mathrm{x}, \mathrm{y}, \mathrm{z})$ plane. It represents the images in sequences and in dynamic manner, which in turn called as video signals.

Here, we are using the digital image processing to transmit signals to traffic signals and thereby triggering the signal to green for ambulances. This can be done by using the placing the motion camera in all traffic signals, which captures the video signals and when there in ambulance in the road, it automatically changes the signal green according to the speed and time where ambulance has been placed. Also, as an improvement of the previous project GPS tracking system will be used this time to track the position of the ambulance. Since, all the traffic signals are monitored and will be automated in the near future; every ambulance will be tracked with the help of the GPS systems. The GPS systems will use the Geographic map information of the area along with the live position data from GPS based vehicle tracking unit installed in the Ambulance, and presents the same on a traffic control station. This will automate the signal to green based on the geographical location of the ambulance. The GPS system can also guide the ambulance to the accident location as well based on the position of the nearest ambulance and accident location. Every ambulance will be attached with a proximity sensor device which emit the signals in particular frequencies to the proximity receiver that has been placed in traffic signals. The receiver that has been placed in the traffic signals will receive the signals transmitted by ambulance based on the approach speed of the ambulance. In this paper, we will be discussing about how the images will be recognized and how the signals will be transmitted and received.

In order to provide the clear image we will be using smoothing filter and sharpening filter which reduce the distortion and interference while capturing the images and in order to provide enhancement for the images for better accoutrement, basic morphological algorithms will be used and for pattern recognition, clustering algorithm is used and for determining shortest path, Djikistra shortest path algorithm will be implemented.

\section{HOW IS THE PROPOSED CONCEPT BETTER THAN BASE CONCEPT?}

Base Paper: The base paper uses image processing technology to capture the images of the ambulance that is approaching the signal. The one drawback that it had was the distance constraint due to the use of RF transmitter and Receiver to generate RF signals in order to enhance the performance of detection of the ambulance presence.

Proposed Concept: The proposed concept too uses image processing technology to capture images. The difference is that here GPS system is used as a replacement for RF transmitter and receiver module in every ambulances. The usage of GPS module overcomes the distance constraint problem and also increases the accuracy of locating the ambulance to the near the traffic signal. The nearest ambulance is located using Djikstra's algorithm.

Also, the idea of using proximity sensors in the proposed concept improves the efficiency and accuracy of tracking the ambulance presence amidst the traffic.

\section{PROPOSED CONCEPT - HOW IT WORKS???}

When the road is completely filled with traffic, it is necessary to have natural traffic signal changes for ambulances that are in very critical situation to save human lives. Here the motion camera that has been placed in traffic signals captures the 3-D Images of vehicles in roads to automatically trigger the signal to green from the distance where the ambulance has been viewed. In order to determine the closest proximity of the ambulance and from which end the ambulance is approaching we have proximity sensor transmitter, that will be placed in ambulances and 
receivers that will be placed in all traffic signals, which calculates the distance and speed of the ambulance to change the signal.

\subsection{Motion Camera}

The motion camera will be placed in the traffic signals which captures the $3 \mathrm{D}$ images in $\mathrm{x}, \mathrm{y}, \mathrm{z}$ plane and determines the location of ambulance and thereby triggers the traffic signal. The unique thing and main use of image capturing is that, all the ambulances will have name AMBULANCE in reverse manner such as 'ECNALUBMA'; this will be used as the primary image recognition pattern.

The motion camera working on Integrated circuits is the major element used in the process of detecting the presence of ambulances. These cameras mainly focus on the parameters like swing angles, actual pan, zooming factor, tilt, and scaled focal length of the camera.

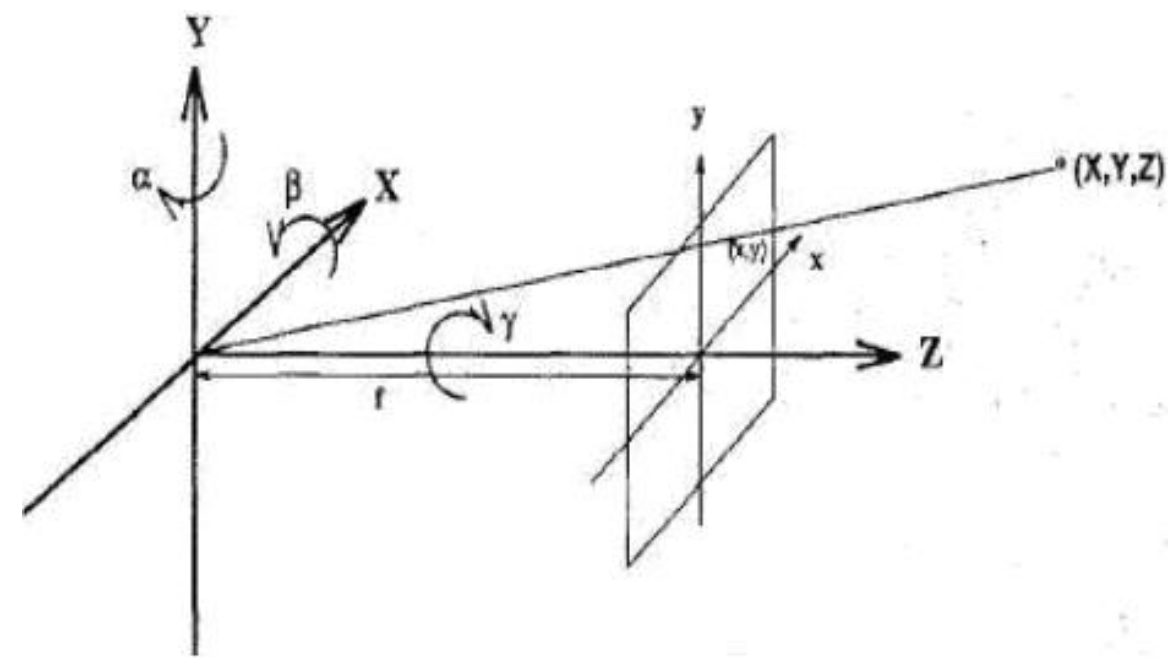

Figure 1: The camera coordinate system, image coordinate system and perspective imaging.

Let $(\mathrm{X}, \mathrm{Y}, \mathrm{Z})$ be considered as a co-ordinate system of the camera. Here, the image plane will be lying perpendicular to $Z$-axis along with its centre which is located at the point $(0,0, f)$. Here, based on perspective projection $\mathrm{P}=(\mathrm{X}, \mathrm{Y}, \mathrm{Z})$ and the $3 \mathrm{D}$ space is projected onto the point, where $\mathrm{p}=(\mathrm{x}, \mathrm{y})$ and the values of $\mathrm{x}$ and $\mathrm{y}$ be $\mathrm{fX} / \mathrm{z}$ and $\mathrm{fY} / \mathrm{z}$ receptively. We will be detracting some of the motion parameters such as,

1. Pan angle a: rotation angle around the $\mathrm{Y}$-axis

2. Zoom factors: ratio of the camera focal lengths between two image frames

3. Tilt angle/3 rotation angle around the $\mathrm{X}$-axis

4. Translation vector $\mathrm{t}=(\mathrm{t}$, ty, $\mathrm{tz}) \mathrm{T} \bullet$

5. Swing angle ry: rotation angle around the Z-axis.

\subsection{Smoothing Filter:}

A smoothing filter is mainly used to replace the each pixel values that are present in input image with its neighbouring images. This in turn, will eliminate the pixel values that are not relevant to its surroundings. It will be like other kernel filters, which considers size and shape of its neighbourhood to calculate sampling. 
The following image will demonstrate the difference between the normal image and smoothing image. We will also be using the Gaussian filter for smoothing the image. Gaussian filter, a bell shaped hump will screen the high spatial frequencies along with the noise and thereby produce the smoothing effect. The distortion in signal will be reduced by smoothing filter, which mainly focuses on the primary image eliminating the unnecessary surroundings.

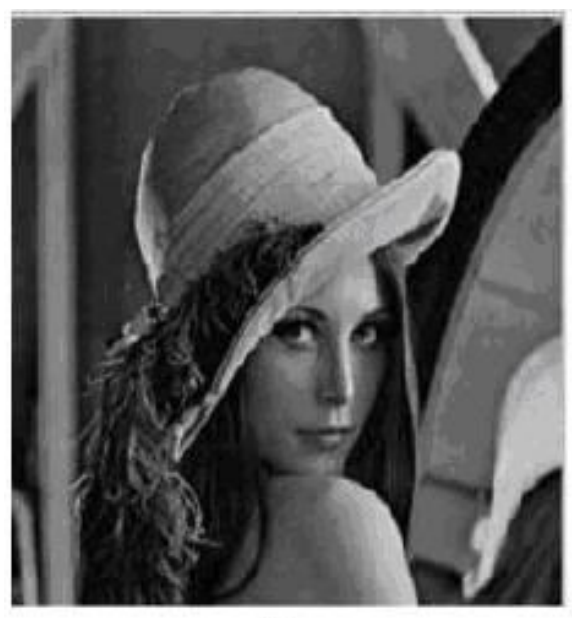

(a)

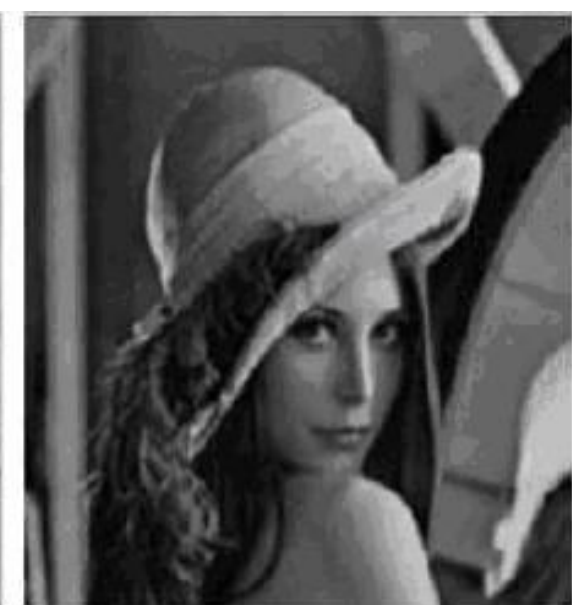

(b)

Fig: a. before smoothing b. after smoothing

The Gaussian filter ID is given as,

$$
G(x)=1 / \sqrt{2 \Pi \sigma} e \frac{-x^{2}}{2 \sigma^{2}}
$$

Where, $\sigma$ is standard deviation of distribution in the Gaussian filter.

\subsection{Sharpening filter:}

The sharpening filter is used to adjust the contrast of the image and also to enhance the edges of objects. Generally, the sharpening filters (High-pass filters) are allowed to pass and delete the low-frequency components. Generally, these sharpening filters will eliminate the noise distortion and provides the proper visibility and image quality, when it is being captured by the camera. The sharpening filters are generally classified as,

1. Derivative filter

2. Laplace filter

3. High pass filter and

4. High boost filter.

Here, we are using the high boost filter to enhance the image further by using low pass.

High boost $=\mathrm{A} *$ original - low pass

$$
\begin{aligned}
& =(\mathrm{A}-1) * \text { original }+(\text { original }- \text { low pass }) \\
& =(\mathrm{A}-1) * \text { original }+ \text { high pass }
\end{aligned}
$$


International Journal of Computer Science \& Information Technology (IJCSIT) Vol 5, No 4, August 2013

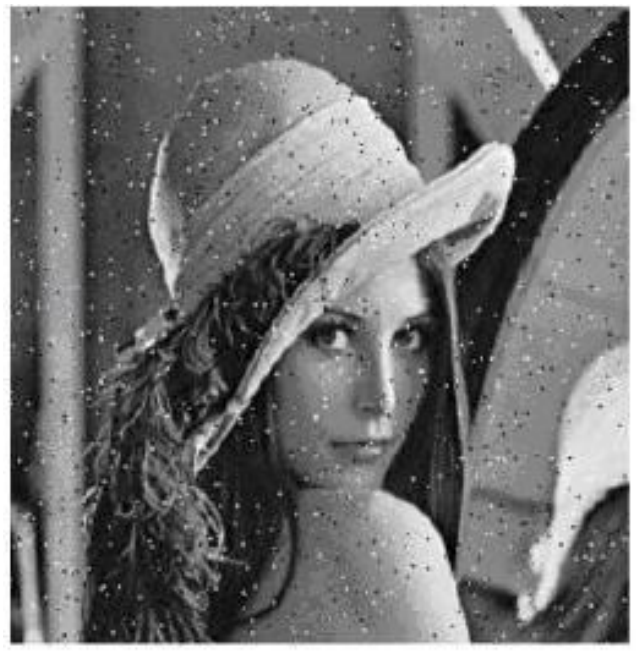

(a)

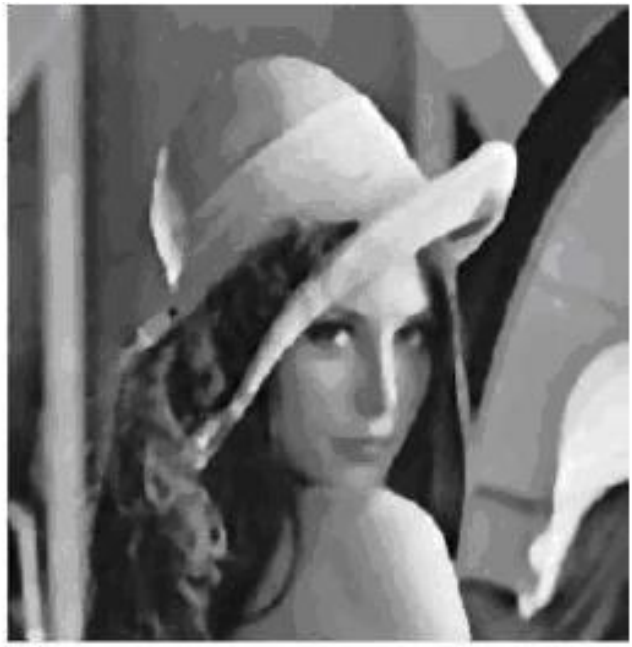

(b)

Fig a) before sharpening b) after sharpening

\subsection{Avoid Image Blur during image capture}

Three types of blur which usually occur in image processing are motion, Gaussian, and compression blurs.

Motion blur maybe due to object movement when a camera shutter remains open for an extended period of time and the object motion within this interval is visible in a single snapshot. It can also be caused by camera movement.

Gaussian blur is made by a soft lens to spread out the light on the focal plane, rather than all going toward a spot. It produces a smoothing effect by removing image details or noises.

[

Compression blur is triggered by the loss of high-frequency components in JPEG compression. The following image describes the above types of image blurs available while capturing an image. 


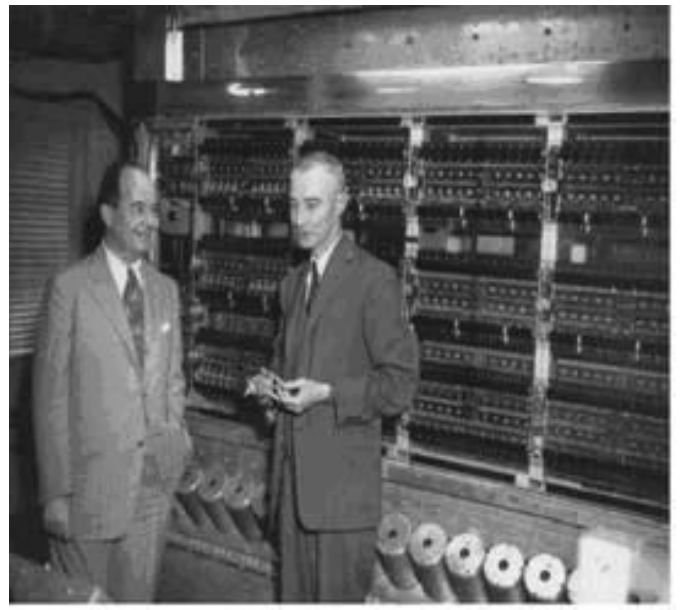

(a)

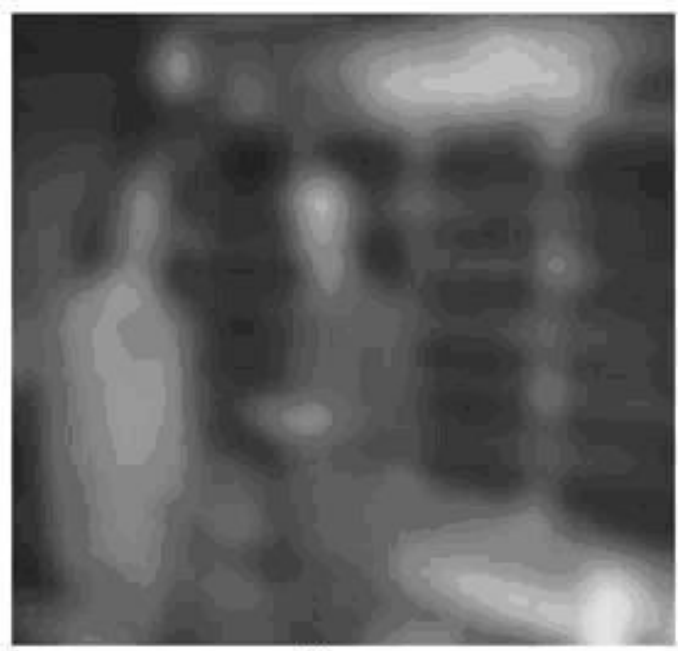

(c)

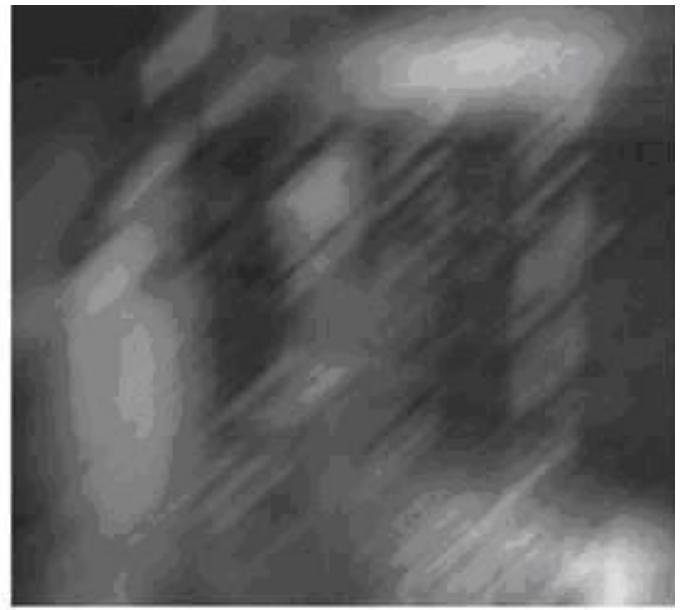

(b)

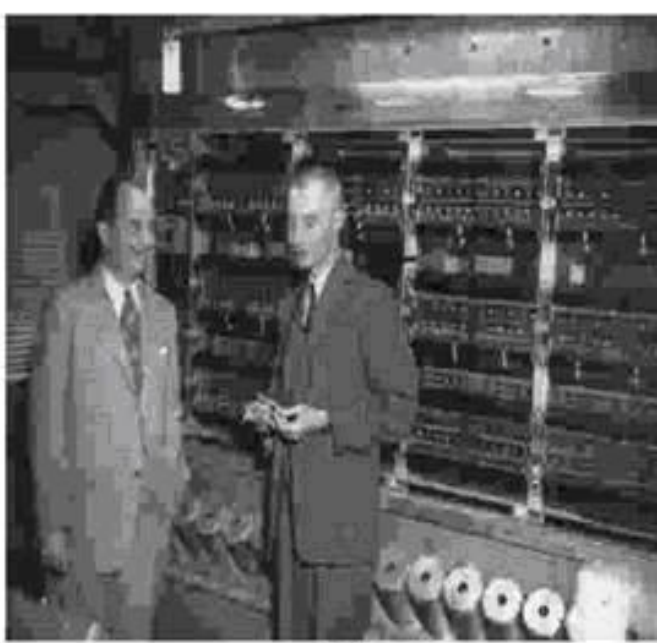

(d)

Fig: (a)Original image, (b)motion blur at orientation 45 degrees and magnitude 40, (c) Gaussian blur with a 17 x 17 window, (d) compression blur with compression ratio of 1:160.

Let $\mathrm{f}\left(\mathrm{x}_{\mathrm{i}}\right)$ denotes the IQM score of an image under the degree of blur $\mathrm{x}_{\mathrm{i}}$. The IQMs used for measuring image blurs must satisfy the monotonically increasing or decreasing property.

(i.e.) if $x_{i+1}>x_{i}$, then $f\left(x_{i+1}\right)-f\left(x_{i}\right)>0$ for monotonically increasing (or) $f\left(x_{i+1}\right)-f\left(x_{i}\right)<0$ for monotonically decreasing property. The sensitivity of IQMs is defined as the score of the aggregate relative distance:

$$
\sum_{i} \frac{f\left(x_{i+1}\right)-f\left(x_{i}\right)}{f\left(x_{i}\right)}
$$

Nine IQMs, which are grouped into three categories based on pixel distance, correlation, and mean square error, are explained as follows,

Let $\mathrm{F}(\mathrm{j}, \mathrm{k})$ denote the pixel value of row $\mathrm{j}$ and column $\mathrm{k}$ in a reference image of size $\mathrm{Mx} \mathrm{N}$, and $\mathrm{F}^{\wedge}(\mathrm{j}, \mathrm{k})$ denote the pixel value in a testing image. 
Category I: IQMs based on pixel distance

1. AD (average distance)

$A D=\sum_{j=1}^{M} \sum_{k=1}^{N} \mid F(j, k)-\hat{F}(j, k) / M N$

2. L2D (L2 Euclidean distance)

$L 2 D=\frac{1}{M N}\left(\sum_{j=1}^{M} \sum_{k=1}^{N}(F(j, k)-\hat{F}(j, k))^{2}\right)^{1 / 2}$

Category II: IQMs based on correlation

3. SC (structure content)

$S C=\sum_{j=1}^{M} \sum_{k=1}^{N} F(j, k)^{2} / \sum_{j=1}^{M} \sum_{k=1}^{N} \hat{F}(j, k)^{2}$

4. IF (image fidelity)

$I F=1-\left(\sum_{j=1}^{M} \sum_{k=1}^{N}(F(j, k)-\hat{F}(j, k))^{2} / \sum_{j=1}^{M} \sum_{k=1}^{N} F(j, k)^{2}\right)$

5. NK (N cross-correlation)

$$
N K=\sum_{j=1}^{M} \sum_{k=1}^{N} F(j, k) \hat{F}(j, k) / \sum_{j=1}^{M} \sum_{k=1}^{N} F(j, k)^{2}
$$

Category III: IQMs based on mean square error

6. NMSE (normal mean square error)

$N M S E=\sum_{j=1}^{M} \sum_{k=1}^{N}(F(j, k)-\hat{F}(j, k))^{2} / \sum_{j=1}^{M} \sum_{k=1}^{N} F(j, k)^{2}$

7. LMSE (least mean square error)

$$
\text { LMSE }=\sum_{j=1}^{M} \sum_{k=1}^{N}(F(j, k)-\hat{F}(j, k))^{2} / \sum_{j=1}^{M} \sum_{k=1}^{N} O(F(j, k))^{2}
$$

8. PMSE (peak mean square error)

$$
P M S E=\frac{1}{M N} \sum_{j=1}^{M} \sum_{k=1}^{N}[F(j, k)-\hat{F}(j, k)]^{2} /\left\{\max _{j, k}[F(j, k)]\right\}^{2}
$$

9. PSNR (peak signal to noise ratio)

$$
P S N R=20 \times \log _{10}\left\{255 /\left\{\sum_{j=1}^{M} \sum_{k=1}^{N}[F(j, k)-\hat{F}(j, k)]^{2}\right\}^{1 / 2}\right.
$$

\subsection{Pattern Recognition}

In order to recognize the pattern of the ambulance in heavy traffic, we will be using the clustering algorithm, in particular known as "two-pass mode clustering algorithm". This algorithm requires and processes only the registered multi-spectral image, twice. Here, the pattern of ambulance will be imparted in the device and whenever the motion camera finds the pattern similar to that of recognized pattern, it will be recognized. In clustering algorithm, there are two passes available. In first pass, the mean vectors of all clusters will be generated and in the next pass, each every 
pixel will be assigned to a cluster that represents a single type, so as to determine the pattern exactly.

The notations that will be used in this algorithm is given below:

B: It represents the total number of bands used. These numbers are the dimensionality in spectral space. Here we are using 3D spectral space.

Cmax: It represents the maximum number of clusters

$\mathbf{r}(\mathbf{P}, \mathbf{k})$ : the distance between mean vector of current cluster $(\mathrm{k})$ and gray value vector of pixel $\mathrm{p}$.

It is given as

$$
r(P, k)=\left(\sum_{i=1}^{B}\left(\operatorname{Mean}_{i}(k)-P_{i}\right)^{2}\right)^{1 / 2}
$$

Where, $1<=\mathrm{k}<=$ Cmax, MEAN denoted the mean value of cluster $\mathrm{k}$ in band $\mathrm{i}$, Pi denotes the gray value in band I of pixel $\mathrm{p}$.

$\mathbf{R}$ is the constant radius in spectral space used to decide the mean vector of new cluster. If the value of $\mathrm{R}$ is lesser then $\mathrm{r}$, then new cluster will be generated.

$\mathbf{d}(\mathbf{k} 1, \mathbf{k} 2)$ represents the difference between mean vector of two different clusters $\mathrm{k} 1$ and $\mathrm{k} 2$, where $1<=\mathrm{k} 1, \mathrm{k} 2<=\mathrm{Cmax}$ and $\mathrm{k} 1 \mathrm{k} 2$.

D is the constant radius in spectral space, which is used to determine whether two distinct clusters $\mathrm{k} 1$ and $\mathrm{k} 2$ should be emerged or not.

$\mathbf{N}$ is the constant that represents the total number of pixels to be evaluated. $\mathrm{n}(\mathrm{k})$ is the total number accumulated in cluster $\mathrm{k}$.

\section{Pass 1: Cluster's Mean vector establishment:}

Here, the gray value vector of first pixel is placed as initial cluster's mean of cluster I. Then Mean is calculated as

$$
\operatorname{MEAN}_{i}(k)_{n e w}=\frac{\operatorname{MEAN}_{i}(k)_{\text {old }} \times n(k) \oplus P_{i}}{n(k) \oplus 1}
$$

If the above equation is used, then the values present in the equation ntotal (total number of pixels) and $\mathrm{n}(\mathrm{k})$ will be incremented by one. This process will be repeated until the new image has been placed. If the value of $\mathrm{K}$ or $\mathrm{N}$ is lesser than $\mathrm{n}$ total and is equal to Cmax, then the process, which has been repeating will be terminated. The distance between the two mean vectors will be used to calculate the recognition pattern. The decision radius finally is mainly dependent upon the condition to activate the clusters and to merge it finally. If the value of $d$ is lesser than or equal to decision radius, then two clusters will be merged and also new mean vector will be determined.

$$
\operatorname{MEAN}_{i}(k)_{n e w}=\frac{\operatorname{MEAN}_{i}\left(k_{1}\right) \times n\left(k_{2}\right)+M E A N_{i}\left(k_{2}\right) \times n\left(k_{2}\right)}{n\left(k_{1}\right) \oplus n\left(k_{2}\right)}
$$


Where, $1<=\mathrm{k}, \mathrm{k} 1, \mathrm{k} 2<=\mathrm{Cmax}$ and also $\mathrm{k} 1 \neq \mathrm{k} 2$. Now, the total number of pixels in the new cluster $\mathrm{n}(\mathrm{k})$ will be sum of the $\mathrm{n}(\mathrm{k} 1)$ and $\mathrm{n}(\mathrm{k} 2)$. At last, the number of clusters are built as the group of individual pixels and its mean vectors will be determined in the second pass

\section{Pass 2: Pixel Classification}

Pass 2 in mainly used to classify each and every pixel into one of the clusters that has been built in pass 1 . The minimum distance method will be adopted here, where the clusters will recognize the images that are present near and by using the minimum distance method, the pixels with spectral features will be merged together, so as to ensure the efficient reliability of the merged images. The pixels will be recognized by the motion camera that has been placed in traffic signals and thereby, the images of ambulance will be clearly recognized by these pass 2 merged image, with the image that has been present closer to the camera will be identified first.

\subsection{Determine the nearest ambulance (Shortest path Algorithm)}

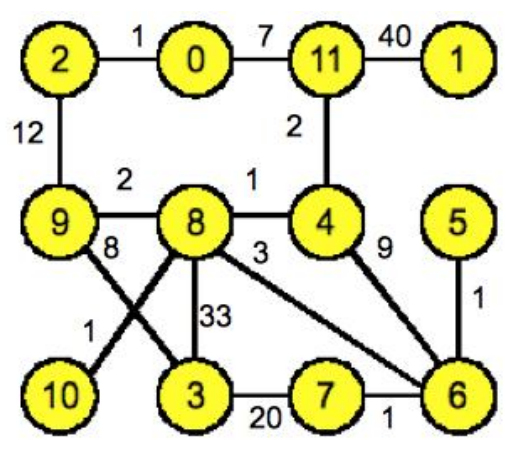

Fig: Djikstra’s Algorithm - Determine the shortest path

The idea behind the shortest path algorithm (Dijkstra's algorithm) is to identify the nearest ambulance in the traffic when there is a case of ambulances coming from more than one side. Since roads come in varying lengths we want to work on weighted graphs for this problem. A weighted graph is simply a graph where each edge e is assigned a non-negative value called the weight, $w(e)$, of the edge. A path is a sequence of vertices $p=\left(v_{1}, \ldots, v_{n}\right)$ such that $v_{i} \sim v_{i+1}$. Set ei $=\left(\mathrm{v}_{\mathrm{i}}, \mathrm{v}_{\mathrm{i}+1}\right)$. The length of a path $\mathrm{p}$ is $\mathrm{d}(\mathrm{p})=\& \operatorname{Sigma} \mathrm{w}\left(\mathrm{e}_{\mathrm{i}}\right)$. For convenience we will also write $\mathrm{w}$ $(\mathrm{u}, \mathrm{v})$ to denote the weight of the edge $(\mathrm{u}, \mathrm{v})$. The idea behind Dijkstra's algorithm is breadth-first search (BFS). This type of search explores vertices by spreading out as new vertices are found.

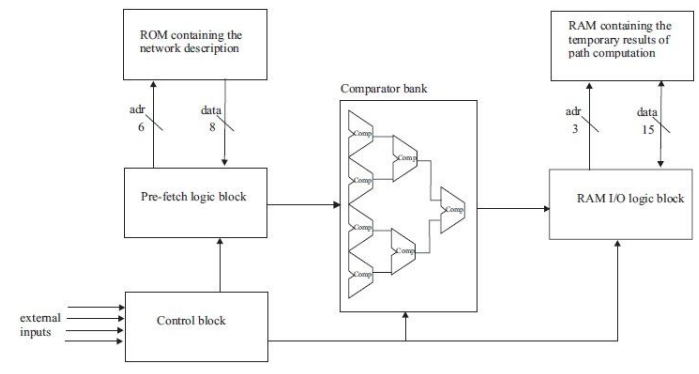

Fig: FPGA-based Dijkstra's shortest pathalgorithm 


\subsection{Why GPS - Global Positioning System replaces RF?}

Global Positioning System tracking is a method of working out exactly where something is. It is a worldwide radio-navigation system formed from the constellation of 24 satellites and their ground stations. A GPS tracking system, for example, may be placed in a vehicle, on a cell phone, or on special GPS devices, which can either be a fixed or portable unit. GPS works by providing information on exact location. It can also track the movement of a vehicle or person. Because of the above flexibility of accessing the modules anywhere across the globe, the biggest disadvantage of distance constraint will be overcome over RF trans receiver modules.

\subsection{How GPS is used here to track the ambulance?}

Every ambulance is provided with a vehicle based tracking unit which will provide the details to the traffic control station. The control station/remote access server location is equipped with a Desktop Computer and a modem corresponding to the particular Radio used for communication between Ambulance and the Control Station. Also, there is a GPS receiver which will keep track of the ambulance position nearest to the signal. The output of the GPS receiver will be helpful in triggering the signal green when the ambulance approaches the signal. Since some control stations have direct control over the traffic signal, the geographical location of the ambulance is also sent to the control station who can act accordingly when an ambulance is nearest to the signal or in case of any emergency.

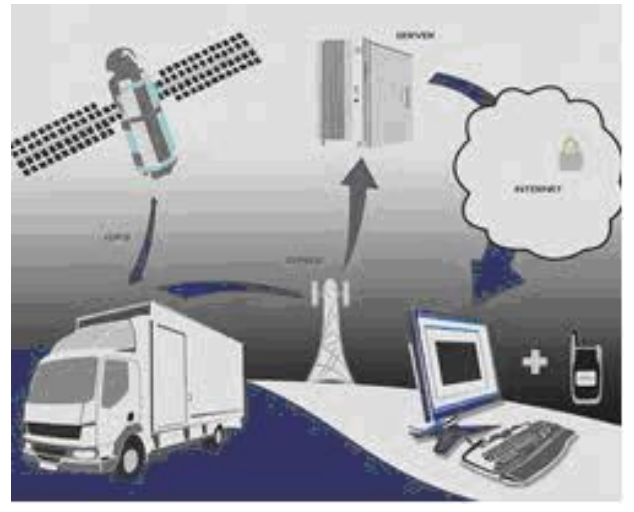

Fig: GPS vehicle tracking system involved in identifying the geographical coordinates of the ambulance.

A vehicle tracking system combines the installation of an electronic device in a vehicle, or fleet of vehicles, with purpose-designed computer software at least at one operational base to enable the owner or a third party to track the vehicle's location, collecting data in the process from the field and deliver it to the base of operation. Modern vehicle tracking systems commonly use GPS technology for locating the vehicle. Vehicle information can be viewed on electronic maps via the Internet or specialized software. Urban public transit authorities/traffic control department personnel are an increasingly common user of vehicle tracking systems, particularly in large cities. So based on the location of the ambulance nearest to the signal, the traffic signal GPS receiver module receives the input data from the transmitter and the signal for that direction is changed to green to allow the ambulance comfortably and swiftly pass the signal.

\subsection{GPS transmitter and Receiver circuits}

The below image shows the simple GPS transmitter and receiver circuit which helps in identifying the exact position of the vehicle (ambulance). 


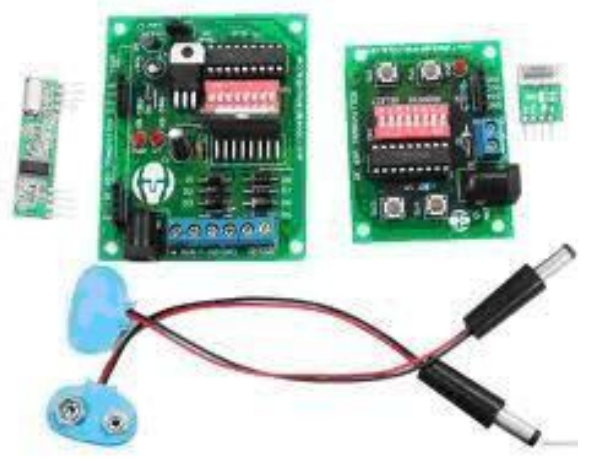

Fig: GPS transmitter receiver circuit

\subsection{Why Proximity sensors?}

The idea of using proximity sensors in the proposed concept improves the efficiency and accuracy of tracking the ambulance presence amidst the traffic. These sensors are sensitive to small changes in the input and this helps in the calculative changing of signal immediately. Every ambulance will be attached with a proximity sensor device which emit the signals in particular frequencies to the proximity receiver that has been placed in traffic signals. The receiver that has been placed in the traffic signals will receive the signals transmitted by ambulance based on the approach speed of the ambulance. These signals will enable the receiver to identify the speed of the ambulance, thereby estimating the distance of the ambulance from the traffic signal. The output of the proximity sensor receiver module is available to processing unit to change the signal to green accordingly.

\section{IMPACT ON HUMAN LIVES}

\section{Current Scenario:}

Now-a-days due to more luxurious living, more four wheelers have replaced 2-wheelers. Moreover, industrialization (tech-parks) and need for space have caused more congestion. This in turn increases traffic. Amidst all these frenzied life, one forgets the importance of human life.

\section{After this work:}

With the implementation of this idea, more lives can be saved. Due to the implementation of this signal generator in every ambulance and image processing techniques, GPS modules, etc., the ambulance would be able to reach the hospital on time which in turn would save a million lives.

\section{FUTURE SCOPE}

The current proposed concept of using GPS to replace the RF transmitter in order to overcome the distance constraint can be extended to alert the nearest ambulance from the control area, to rush the ambulance to the accident spot and provide the help to the injured person(s).

\section{CONCLUSION}

Thus the real time implementation of proposed concept of implementing GPS modules in ambulances and image processing technology in traffic rich areas could ease the difficulties faced during jams by ambulances when rushing to the hospital or to the injured spot. 


\section{REFERENCES}

[1] Manoj Prabahkar, Manoj Kumar \& Dhilip Narayan (May 2013), "IMPACT OF IMAGE PROCESSING IN SAVING THE HUMAN LIFE BY AUTOMATING TRAFFIC SIGNALS" published in IJSRN, Volume 1 Issue 3.

[2] FRANK Y. SHIH, "IMAGE PROCESSING AND PATTERN RECOGNITION - Fundamentals and Techniques" A JOHN WILEY \& SONS, INC., Pubilication, Copyrights 2010

[3] STrobotix, Chandigarh labs and Chawla Radios, "RF based wireless remote"

[4] Yoshihiro Ikefuji, Sadakazu Murakami"Video Signal Generator circuit and video image processing device using the same", Patent Number - 5311296, Rohm Co., Ltd., May10, 1994

[5] Yap-Peng Tan, Sanjeev R. Kulkarni, Peter J. Ramadge, "A NEW METHOD FOR CAMERA MOTION PARAMETER ESTIMATION"

[6] BRB900 GPS Telemetry System May 10, 2011, Version 0.05.

[7] http://eoinbailey.com/blog/dijkstras-algorithm-illustrated-explanation

[8 ]http://www.articlesbase.com/technology-articles/advantages-disadvantages-and-applications-ofmotion-capture-217465.html

[9] http://www.cs.princeton.edu/ rs/AlgsDS07/15ShortestPaths

[10] https://www.linxtechnologies.com/en/products/modules/lr-rf-transmitter-receiver

\section{Authors}

Manoj Prabhakar is a Systems Engineer in Tata Consultancy Services. He pursued the Bachelor of Technology in Information Technology in Anna University in Chennai and now pursuing Master of Business Administration in Anna University. His researches have been mainly inclined towards the development of nation with some social factors included in it. His other projects are also based on widely spread social issues and suggesting a solution for it. He is also a part of Peace and Humanity organization to support people. He has also worked in Marketing Research Management of CITIBANK N.A

Manoj Kumar is a Systems Engineer in Tata Consultancy Services. He pursued the Bachelor of Technology in Information Technology in Anna University in Chennai and now pursuing Master of Business Administration in Anna University. He is currently working Health Care Project of CITIBANK N.A
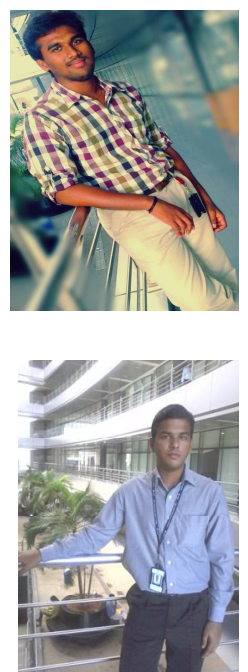